\title{
ON TWO-SIDED ESTIMATE FOR NORM OF FOURIER OPERATOR
}

\section{I.A. SHAKIROV}

\begin{abstract}
In the work we study the behavior of Lebesgue constant $L_{n}$ of the Fourier operator defined in the space of continuous $2 \pi$-periodic functions. The known integral representations expressed in terms of the improper integrals are too cumbersome. They are complicated both for theoretical and practical purposes.

We obtain a new integral representation for $L_{n}$ as a sum of Riemann integrals defined on bounded converging domains. We establish equivalent integral representations and provide strict two-sided estimates for their components. Then we provide a two-sided estimate for the Lebesgue constant. We solve completely the problem on the upper bound of the constant $L_{n}$. We improve its known lower bound.
\end{abstract}

Keywords: partial sums of Fourier series, norm of Fourier operator, Lebesgue constant, asymptotic formula, estimate for Lebesgue constant, extremal problem.

Mathematics Subject Classification: 34A25, 22E05

\section{INTRODUCTION}

If the Fourier series of a continuous $2 \pi$-periodic function $x=x(t)$ is its uniformly convergent expansion, then the partial sums of this series

$$
S_{n} x(t)=\frac{1}{\pi} \int_{0}^{2 \pi} x(s) D_{n}(t-s) d s, \quad D_{n}(t)=\frac{\sin \left(n+\frac{1}{2}\right) t}{2 \sin \frac{t}{2}}
$$

serve as an approximate expression for the initial function [1], [2]. The Fourier operator

$$
S_{n}: B \mapsto H_{n}^{T} \subset B, \quad B=C[0,2 \pi] \text { or } \quad B=L_{1}(0,2 \pi),
$$

corresponding to polynomial (1) and acting in $B$ has the minimal norm ([3], [4]) among all projectors

$$
P_{n}: B \mapsto H_{n}^{T} \subset B, \quad H_{n}^{T}=\left\{T_{n}(t) \mid T_{n}(t)=a_{0}+\sum_{k=1}^{n}\left(a_{k} \cos k t+b_{k} \sin k t\right)\right\} .
$$

In other words, for each natural $n$ the inequality

$$
\left\|P_{n}\right\|_{B} \geqslant\left\|S_{n}\right\|_{B} \equiv L_{n}
$$

holds. According this inequality, among the mentioned projectors, the norm of operator (2) deserves the most attention. The quantity $L_{n}$ is called Lebesgue constant; it satisfies the formula

$$
L_{n}=\frac{1}{\pi} \int_{0}^{2 \pi}\left|D_{n}(t)\right| d t=\frac{2}{\pi} \int_{0}^{\frac{\pi}{2}} \frac{|\sin (2 n+1) t|}{\sin t} d t, \quad n \in \mathbb{N} .
$$

I.A. Shakirov, On two-Sided ESTIMATE FOR NORM OF Fourier OPERATOR.

(c) SHAKIROV I.A. 2018.

Submitted July 14, 2016. 
This is the main characteristics of approximating initial function by polynomials (1) involved in estimating the error of the approximation in the Lebesgue inequality (fundamental inequality):

$$
\left\|x-S_{n} x\right\|_{B} \leqslant\left(1+L_{n}\right) E_{n}^{T}(x), \quad x \in B, \quad n \in \mathbb{N},
$$

where $E_{n}^{T}(x)$ is the best possible approximation of the function $x(t)$ by trigonometric polynomials of form $T_{n}(t)$; the identity $n=0$ corresponds to the trivial approximation.

The properties of operator (2) and of its generalizations and the corresponding fundamental characteristics were studied in great details by A. Lebesgue, L. Fejér, G. Hardy, J. Littlewood, G. Szegö, A. Zygmund, J. Marcinkiewicz and others. An essential contribution was made by Soviet mathematicians S.N. Bernstejn, A.N. Kolmogorov, N.P. Korneichuk, S.M. Nikolskii, I.P. Natanson, A.I. Stepanets, S.B. Stechkin, Yu.N. Subbotin, P.K. Suetin, A.F. Timan, M.F. Timan, V.M. Tikhomirov, S.A. Telyakovskii, their numberous pupils and followers.

In the beginning of the previous centenary, L. Fejér established an asymptotic identity for constant (3) [5]:

$$
L_{n}=\frac{4}{\pi^{2}} \ln n+O(1), \quad n \rightarrow \infty .
$$

Then he in [6] and G. Szegö in [7] found the formulae for calculating exact value of constant (3):

$$
L_{n}=\frac{1}{2 n+1}+\frac{2}{\pi} \sum_{k=1}^{n} \frac{1}{k} \tan \frac{\pi k}{2 n+1}, \quad L_{n}=\frac{16}{\pi^{2}} \sum_{k=1}^{\infty} \frac{1}{4 k^{2}-1} \sum_{m=1}^{k(2 n+1)} \frac{1}{2 m-1}, \quad n \in \mathbb{N}, \quad L_{0}=1 .
$$

More detailed information on other representations for constant (3), on its generalizations $L_{\frac{n}{2}}$ and on their properties can be found in monographs [2], [9], [14] and in works [7], [8]. The issues on upper bounds for $L_{n}$ were considered many times in mathematical literature and less often on lower bounds, see [1]-[4], [8]-[15]. For instance, in monograph [14], for the difference

$$
O(n) \equiv L_{n}-\frac{4}{\pi^{2}} \ln n, \quad n \in \mathbb{N},
$$

there was provided the two-sided inequality $\frac{1}{3}<O(n)<3$. In work [13], on the base of a result by G. Watson [8], P.V. Galkin obtained the two-sided inequality

$$
1 \leqslant L_{n}-\frac{4}{\pi^{2}} \ln (n+1)<1.8724, \quad n=0,1,2, \ldots
$$

A technical mistake in upper bound in (6) made in work [15] was corrected to a smaller quantity

$$
c_{0}+\frac{4}{\pi^{2}} \ln 2 \approx 1.2706, \quad c_{0}=\frac{8}{\pi^{2}} \sum_{k=1}^{\infty} \frac{\ln k}{4 k^{2}-1}+\ln 2+\frac{\gamma}{2} \approx 0.9897
$$

where $\gamma$ is the Euler constant. In another work [16] by G.I. Natanson, the constant $c_{0}$ was specified; this constant plays a principal role while studying constant (3). In Lemma 2 in [16], in order to improve known estimates for the Lebesgue constants of Vallée Poussin sums, there were introduced more general continuous analogues of Lebesgue constants $L(n), n \in[1, \infty)$. We note that for the natural $n$, the results obtained in this lemma are in agreement with inequalities (4), (5) and their generalizations (6), (7) in work [13]. In Remark 2 in [16] there was noted that it is interesting to know the behaviour of the difference

$$
O(n, a) \equiv L_{n}-\frac{4}{\pi^{2}} \ln (n+a), \quad a \in[0,+\infty),
$$

as a function of two variables; here the differences $O(n, a)$ corresponding to different values of the parameter $a$ behave differently (strictly increase or decrease, have different domains). For 
instance, it was established in [13] that the difference $L_{\frac{n}{2}}-\frac{4}{\pi^{2}} \ln (n+1)$ strictly decreases in $n$, and the difference $L_{\frac{n}{2}}-\frac{4}{\pi^{2}} \ln (n+2)$ strictly increases.

In the framework of the present work we study the classical version of the difference

$$
O_{n} \equiv O(n, 0)=L_{n}-\frac{4}{\pi^{2}} \ln n, \quad n \in \mathbb{N},
$$

corresponding to the shift $a=0$ in the argument of the logarithmic function in (7).

For constant (3), in work [10], G. Hardy obtained the integral formulae:

$$
L_{n}=4 \int_{0}^{\infty} \frac{\tanh (2 n+1) t}{\tanh t} \frac{d t}{\pi^{2}+4 t^{2}}, \quad L_{n}=\frac{4}{\pi^{2}} \int_{0}^{\infty} \frac{\sinh (2 n+1) t}{\sinh t} \ln \left(\operatorname{coth}\left(n+\frac{1}{2}\right) t\right) d t
$$

involving no absolute value in the integrand. They are complicated for theoretical studies and approximate calculations since they are expressed via improper integrals of very complicated rational hyperbolic and hyperbolic logarithmic functions. Therefore, it is of interest to find simpler integral formulae for $L_{n}$. These issues are considered in details in Section 3.

The researches in this direction are still active nowadays. In works [17]-[20], the problems on approximations of periodic and non-periodic functions by various orthogonal polynomials (Fourier-Legendre, Fourier-Jacobi, Fourier-Chebyshev, etc.) are solved in weighted and generalized weighted spaces. In these papers, a special attention is paid to obtaining two-sided estimates for corresponding fundamental characteristics, the sharpness of the Lebesgue inequality, as well as to studying approximative properties of some modifications of partial Fourier sums on various classes of functions. In the case of Lagrange interpolation and sinc-approximations of functions, similar problems were solved in works by the authors [21]-[23].

In the present work we obtain the following new results:

1) employing specific nodes, we obtain a new integral representation for $L_{n}$ expressed via Riemann integrals of trigonometric functions with the shift of the independent variable over domain narrowing as the parameter $n$ grows; we provide equivalent integral representations;

2) we define sharper than above two-sided estimates for the difference $O_{n}$;

3) on the base of the obtained results we solve the extremal problem

$$
\inf \left\{A \in \mathbb{R}^{+} \mid L_{n} \leqslant A+\frac{4}{\pi^{2}} \ln n \quad \forall n \in \mathbb{N}\right\}=A^{*}, \quad A^{*}=\frac{1}{3}+\frac{2 \sqrt{3}}{\pi}=1.435991 \ldots
$$

\section{Auxiliary Statements}

In more detailed studying the properties of Lebesgue constant (3) and in the proofs of the most part of lemmata and theorems in the work we shall need functional classes $V_{\delta}^{ \pm}$.

Definition 1. A strictly monotone function $\varphi=\varphi(n)(n \in D=D(\varphi) \subseteq \mathbb{N})$ of a discrete variable $n$ belongs to one of the classes $V_{\delta}^{ \pm}$if the variation

$$
\delta_{\varphi} \equiv \delta(\varphi)=\sup \{\varphi(n) \mid n \in D\}-\inf \{\varphi(n) \mid n \in D\}, \quad \delta_{\varphi}>0,
$$

of its range $R(\varphi)$ obeys the condition $\delta_{\varphi}<\delta$, where $\delta$ is some number; the sign ' + ' in the notation $V_{\delta}^{ \pm}$is used if the function increases in the domain $D$ and the sign '-' is chosen if it decreases.

It is clear that these classes are defined as the family of the functions $\{\varphi\}$, whose variations satisfy the inequality $\delta_{\varphi}<\delta$ (in our case $\delta=0.2$ ). The functions involved in lemmata and theorems in this work have a very small variation. Even the "worst" function $\bar{\varphi}=\bar{\varphi}(n), n \in D$, satisfies the inequality $\delta_{\bar{\varphi}}<0.2$. 
Remark 1. For continuous continuations $\bar{\varphi}=\bar{\varphi}(n)(n \in \bar{D}=\overline{(\inf D ; \sup D)} \subset \mathbb{R})$ of discrete functions $\varphi=\varphi(n)(n \in D \subset \mathbb{N})$ the formulation and matter of Definition 1 are kept true.

Remark 2. The functions (sequences) in the classes $V_{\delta}^{+}$and $V_{\delta}^{-}$possess a remarkable property that their greatest oscillations correspond to the initial values of the variable $n$ ( $n=1$ or $n=1,2$ or $n=1,2,3)$ followed by a "stabilization" at certain limiting points. This property is employed in the work for obtaining more gentle estimates for various integral representations of the Lebesgue constant.

Let us provide auxiliary lemmata needed in what follows.

Lemma 1. The function

$$
\alpha_{n} \equiv \alpha(n)=\frac{1}{(2 n+1) \sin \frac{\pi}{4 n+2}}, \quad \alpha: D \mapsto \mathbb{R}, \quad D=D_{1} \quad \text { or } \quad D=D_{2}, \quad D \subset \mathbb{N},
$$

and its linear combination

$$
\varphi_{1}=\varphi_{1}(n)=\frac{\pi}{2} \theta \alpha_{n}, \quad \theta=\frac{2}{\pi} \operatorname{Si}\left(\frac{\pi}{2}\right), \quad \operatorname{Si}(x)=\int_{0}^{x} \frac{\sin t}{t} d t
$$

are strictly increasing functions of the discrete variable $n$.

Proof. We represent function (9) as

$$
\alpha_{n}=\frac{2}{\pi}\left(\frac{\sin \frac{\pi}{4 n+2}}{\frac{\pi}{4 n+2}}\right)^{-1}, \quad n \in \mathbb{N} .
$$

The sequence

$$
\tilde{\alpha}_{n}=\frac{\sin \frac{\pi}{4 n+2}}{\frac{\pi}{4 n+2}}
$$

increases monotonically, is bounded and it satisfies the relations

$$
\frac{3}{\pi} \leqslant \tilde{\alpha}_{n}<1, \quad \lim _{n \rightarrow \infty} \tilde{\alpha}_{n}=1
$$

which are implied easily by the known properties of the limit

$$
\lim _{x \rightarrow 0} \frac{\sin x}{x}=1
$$

At that, the initial sequence $\alpha_{n}$ decreases monotonically and has the following properties:

$$
\begin{aligned}
& n \in D_{1} \Rightarrow R\left(\alpha_{n}\right)=\left(\frac{2}{\pi}, \frac{2}{3}\right] \subset(0.636619,0.666667), \\
& \delta\left(\alpha_{n}\right)=\frac{2}{3}-\frac{2}{\pi}=0.03004 \ldots, \\
& n \in D_{2} \Rightarrow R\left(\alpha_{n}\right)=\left(\frac{2}{\pi}, \frac{1}{7 \sin \frac{\pi}{14}}\right] \subset(0.636619,0.641995), \\
& \delta\left(\alpha_{n}\right)=\frac{1}{7 \sin \frac{\pi}{14}}-\frac{2}{\pi}=0.00537 \ldots
\end{aligned}
$$

It is clear that the multiplication of the sequence $\alpha_{n}$ by a constant $\frac{\pi}{2} \theta, \theta=0.872654 \ldots$, preserves its monotonicity and only the range and variation slightly change:

$$
D_{1}=\mathbb{N} \quad \Rightarrow \quad R\left(\varphi_{1}\right)=\left(\theta, \frac{\pi}{3} \theta\right] \subset(0.872654,0.913842), \quad \delta\left(\varphi_{1}\right)=0.04118 \ldots
$$




$$
\begin{aligned}
& D_{2}=\{3,4,5,6, \ldots\} \Rightarrow R\left(\varphi_{1}\right)=\left(\theta, \frac{\pi \theta}{14 \sin \frac{\pi}{14}}\right] \subset(0.872654,0.880022), \\
& \delta\left(\varphi_{1}\right)=0.00736 \ldots
\end{aligned}
$$

Relations (12), (13) for function (10) (as well as (11) for function (9)) are established on the base of simple calculations. Therefore, the functions $\alpha(n), \varphi_{1}(n)$ belong to the class $V_{\delta}^{-}, \delta=0.2$. The proof is complete.

To shorten the notation, hereafter we omit the chosen value $\delta=0.2$ in the notations of the classes $V_{\delta}^{+}, V_{\delta}^{-}$, see the comments after Definition 1 .

Lemma 2. The function

$$
\varphi_{2}=\varphi_{2}(n)=\frac{4}{\pi^{2}} \ln \left(\left(2+\frac{1}{n}\right) \alpha_{n}\left(1+\cos \frac{\pi}{4 n+2}\right)\right)
$$

satisfies the relations

$$
\begin{aligned}
& n \in D_{1} \Rightarrow R\left(\varphi_{2}\right)=\left(\frac{4}{\pi^{2}} \ln \frac{8}{\pi}, \frac{4}{\pi^{2}} \ln (2+\sqrt{3})\right] \subset(0.378824,0.533743), \\
& \delta\left(\varphi_{2}\right)=0.15491 \ldots, \\
& n \in D_{2} \Rightarrow R\left(\varphi_{2}\right)=\left(\frac{4}{\pi^{2}} \ln \frac{8}{\pi}, \frac{4}{\pi^{2}} \ln \frac{\operatorname{cosec} \frac{\pi}{14}+\cot \frac{\pi}{14}}{3}\right] \subset(0.378824,0.439594), \\
& \delta\left(\varphi_{2}\right)=0.06077 \ldots,
\end{aligned}
$$

and belongs to the class $V_{\delta}^{-}$.

Proof. We represent the expression under the logarithm in (14) as the sum of two positive functions, that is, in the form:

$$
\left(2+\frac{1}{n}\right) \alpha_{n}+\left(2+\frac{1}{n}\right) \alpha_{n} \cos \frac{\pi}{4 n+2} .
$$

The first of them belongs to the class $V_{\delta}^{-}$as a product of two functions in the same class, the second also belongs to $V_{\delta}^{-}$according Lemma 2 in [24]. Therefore, the sum and its logarithm also belong to the class $V_{\delta}^{-}$since logarithm does not break the monotonicity. Relations (15) and (16) can be justified by simple calculations. The proof is complete.

Lemma 3. The functions

$$
\begin{aligned}
\varphi_{3} & =\varphi_{3}(n)=\frac{2}{\pi} \alpha_{n}^{2} \cos \frac{\pi}{4 n+2}, \quad n \in D, \quad D=D_{1} \quad \text { or } \quad D=D_{2}, \\
\varphi_{4} & =\varphi_{4}(n)=\frac{2}{\pi}\left(1-\frac{2}{\pi}\right) \alpha_{n}^{2} \cos \frac{\pi}{4 n+2}, \quad n \in D,
\end{aligned}
$$

expressed via (9) are strictly increasing in their domains. They satisfy the relations:

$$
\begin{aligned}
& n \in D_{1} \Rightarrow R\left(\varphi_{3}\right)=\left[\frac{4 \sqrt{3}}{9 \pi}, \frac{8}{\pi^{3}}\right) \subset(0.245035,0.258013), \quad \delta\left(\varphi_{3}\right)=0.01297 \ldots, \\
& n \in D_{2} \Rightarrow R\left(\varphi_{3}\right)=\left[\frac{2}{49 \pi} \operatorname{cosec} \frac{\pi}{14} \cot \frac{\pi}{14}, \frac{8}{\pi^{3}}\right) \subset(0.255808,0.258013), \\
& \delta\left(\varphi_{3}\right)=0.00220 \ldots, \\
& n \in D_{1} \Rightarrow R\left(\varphi_{4}\right)=\left[\frac{4 \sqrt{3}}{9 \pi}\left(1-\frac{2}{\pi}\right), \frac{8}{\pi^{3}}\left(1-\frac{2}{\pi}\right)\right) \subset(0.089040,0.093757), \\
& \delta\left(\varphi_{4}\right)=0.00471 \ldots,
\end{aligned}
$$




$$
\begin{aligned}
n \in D_{2} \Rightarrow R\left(\varphi_{4}\right)= & {\left[\frac{2}{49 \pi}\left(1-\frac{2}{\pi}\right) \operatorname{cosec} \frac{\pi}{14} \cot \frac{\pi}{14}, \frac{8}{\pi^{3}}\left(1-\frac{2}{\pi}\right)\right) } \\
& \subset(0.092955,0.093757), \quad \delta\left(\varphi_{4}\right)=0.00080 \ldots,
\end{aligned}
$$

that is, they belong to the class $V_{\delta}^{+}$.

Proof. In order to study the function $\varphi_{3}(n)$ by means of the derivative, we extend it continuously for a non-discrete domain $\bar{D}=\bar{D}_{1}$ or $\bar{D}=\bar{D}_{2}, \bar{D}_{1}=[1,+\infty), \bar{D}_{2}=[3,+\infty)$. This is a smooth function, that is, $\varphi_{3} \in C^{1}(\bar{D})$. Let us calculate its derivative:

$$
\begin{aligned}
\varphi_{3}^{\prime}(n) & =\frac{2}{\pi}\left(2 \alpha_{n} \alpha_{n}^{\prime} \cos \frac{\pi}{4 n+2}+\frac{\pi}{(2 n+1)^{2}} \alpha_{n}^{2} \sin \frac{\pi}{4 n+2}\right) \\
& =\frac{2}{\pi} \alpha_{n}\left(2 \frac{\pi \cos \frac{\pi}{4 n+2}-(4 n+2) \sin \frac{\pi}{4 n+2}}{(2 n+1)^{3} \sin ^{2} \frac{\pi}{4 n+2}} \cos \frac{\pi}{4 n+2}+\frac{\pi}{(2 n+1)^{2}} \alpha_{n} \sin \frac{\pi}{4 n+2}\right) \\
& =\frac{2}{\pi(2 n+1)} \alpha_{n}^{3}\left(2 \pi \cos ^{2} \frac{\pi}{4 n+2}-2(4 n+2) \sin \frac{\pi}{4 n+2} \cos \frac{\pi}{4 n+2}+\pi \sin ^{2} \frac{\pi}{4 n+2}\right) \\
& =\frac{2}{\pi(2 n+1)} \alpha_{n}^{3}\left(2 \pi-\pi \sin ^{2} \frac{\pi}{4 n+2}-2(2 n+1) \sin \frac{\pi}{4 n+2}\right) \\
& =\frac{2 \alpha_{n}^{3}}{\pi(2 n+1)}\left(\frac{3}{2} \pi+\frac{\pi}{2} \cos \frac{\pi}{2 n+1}-(4 n+2) \sin \frac{\pi}{2 n+1}\right) \\
& =\frac{4 \alpha_{n}^{3}}{2 n+1}\left(\frac{3}{4}+\frac{1}{4} \cos \frac{\pi}{2 n+1}-\frac{\sin \frac{\pi}{2 n+1}}{2 n+1}\right) \\
& =\frac{4 \alpha_{n}^{3}}{2 n+1}\left(\frac{\pi^{2}}{4 !(2 n+1)^{2}}+\frac{1}{4 !}\left(\frac{1}{4}-\frac{1}{5}\right)\left(\frac{\pi}{2 n+1}\right)^{4}\right. \\
& \left.-\frac{1}{6 !}\left(\frac{1}{4}-\frac{1}{7}\right)\left(\frac{\pi}{2 n+1}\right)^{6}+\frac{1}{8 !}\left(\frac{1}{4}-\frac{1}{9}\right)\left(\frac{\pi}{2 n+1}\right)^{8}-\ldots\right) .
\end{aligned}
$$

The obtained Leibnitz series is positive for all $n$ and this is why $\varphi_{3}^{\prime}(n)>0$ for each $n \in \bar{D} \subset \mathbb{R}$. The functions $\varphi_{3}(n)$ and $\varphi_{4}(n)$ differ just by a constant and this is why $\varphi_{4}^{\prime}(n)>0$ for each $n \in \bar{D} \subset \mathbb{R}$. Therefore, functions (17) and (18) strictly increase in the domain $\bar{D}$. Relations (19)-(22) for their range and variations can be proved easily.

In view of Remark 1 we can state that $\varphi_{3}, \varphi_{4} \in V_{\delta}^{+}$. The proof is complete.

While estimating from below the Lebesgue constant in Section 4, we shall employ the functions in the class $V_{\delta}^{-}$and it is sufficient to study their behavior in the domain $D_{1}=\mathbb{N}$. The needed information is provided in the following lemma.

Lemma 4. The functions of the discrete variable

$$
\varphi_{5}(n)=\frac{4}{\pi^{2}} \ln \left(\left(2+\frac{1}{n}\right) \alpha_{n} \cos \frac{\pi}{4 n+2}\right), \quad \varphi_{6}(n)=\frac{1}{\pi} \alpha_{n} \sec \frac{\pi}{4 n+2}, \quad n \in \mathbb{N},
$$

are strictly increasing in $\mathbb{N}$. Their ranges and variations satisfy the relations

$$
\begin{aligned}
& R\left(\varphi_{5}\right)=\left(\frac{4}{\pi^{2}} \ln \frac{4}{\pi}, \frac{2}{\pi^{2}} \ln 3\right] \subset(0.097902394,0.222625359), \quad \delta\left(\varphi_{5}\right)=0.12472 \ldots, \\
& R\left(\varphi_{6}\right)=\left(\frac{2}{\pi^{2}}, \frac{4 \sqrt{3}}{9 \pi}\right] \subset(0.202642367,0.245035065), \quad \delta\left(\varphi_{6}\right)=0.04239 \ldots
\end{aligned}
$$


Proof. The sequence

$$
\left(2+\frac{1}{n}\right) \alpha_{n} \cos \frac{\pi}{4 n+2}, \quad n \in \mathbb{N},
$$

is strictly decreasing (see Lemma 2 in [24]) and logarithm and multiplication by a constant does not break this property. Its range and variation satisfy the relations in Lemma 4, which are obtained in view of the monotonicity of the aforementioned transforms.

The products $\alpha_{n}$ and $\sec \frac{\pi}{4 n+2}$ involved in $\varphi_{6}(n), n \in \mathbb{N}$, are strictly decreasing functions. The relations for the range $R\left(\varphi_{6}\right)$ and for the variation $\delta\left(\varphi_{6}\right)$ are established on the base of simple calculations.

Therefore, $\varphi_{5}, \varphi_{6} \in V_{\delta}^{-}$and this completes the proof.

\section{INTEGRAL REPRESENTATIONS FOR LEBESGUE CONSTANT}

In a more detailed studying of fundamental characteristic (functions and Lebesgue constants) of trigonometric interpolation Lagrange polynomials, an important role is played by the choice of the interpolation nodes, which determines then [24] their various explicit (absolute value free) forms. In our case, while transforming formula (3), a proper choice of the nodes also has a primary importance and it allows to get rid of the absolute value in the integrand in formula (3) and to obtain a new integral representation for the Lebesgue constant.

Theorem 1. Constant (3) satisfies the formula

$$
L_{n}=I_{0}(n)+I(n), \quad L_{n}=L(n), \quad n \in \mathbb{N},
$$

where

$$
\begin{aligned}
I_{0}(n) & =\frac{2}{\pi} \int_{0}^{T} \frac{\sin (2 n+1) t}{\sin t} d t, \quad T=t_{1}=\frac{\pi}{2(2 n+1)}, \quad n \in \mathbb{N}, \\
I(n) & =\frac{2}{\pi} \sum_{k=1}^{n} \int_{0}^{T}\left(\frac{\cos (2 n+1) t}{\sin \left(t+t_{2 k-1}\right)}+\frac{\sin (2 n+1) t}{\sin \left(t+t_{2 k}\right)}\right) d t \\
& =\frac{2}{\pi} \sum_{k=1}^{n} \int_{0}^{T} \frac{\cos (2 n+1) t}{\sin \left(t+t_{2 k-1}\right)} d t+\frac{2}{\pi} \sum_{k=1}^{n} \int_{0}^{T} \frac{\sin (2 n+1) t}{\sin \left(t+t_{2 k}\right)} d t, \\
t_{2 k-1} & =\frac{\pi(2 k-1)}{4 n+2}, \quad k=1, \ldots, n, \quad t_{2 k}=\frac{2 \pi k}{4 n+2}, \quad k=1, \ldots, n .
\end{aligned}
$$

Proof. In the segment $\left[0, \frac{\pi}{2}\right]$ we consider the system of nodes $t_{j}=\frac{\pi}{2(2 n+1)} j, j=0, \ldots, 2 n+1$, formed by the zeroes and extrema of the function $y=|\sin (2 n+1) t|, t \in\left[0, \frac{\pi}{2}\right]$. This system partitions the considered segment into $N=2 n+1$ same parts. Skipping the boundary nodes, we split the rest into two subclasses: $t_{2 k-1}=\frac{\pi}{4 n+2}(2 k-1), k=1, \ldots, n$, which are the maxima of the considered function in the interval $\left(0, \frac{\pi}{2}\right)$, and $t_{2 k}=\frac{2 \pi k}{4 n+2}=\frac{\pi}{2 n+1} k, k=1, \ldots, n$, which are its zeroes in the same domain.

Then we represent Lebesgue constant (3) as the sum of $N$ integrals and we change a variable in each of the integrals. The appropriate new variables $u=t-t_{2 k-1}, v=t-t_{2 k}, u, v \in[0, T]$, $k=1, \ldots, n$, allow us to get rid of the absolute value in formula (3) and to obtain representation $(23)$ :

$$
L_{n}=\frac{2}{\pi} \int_{0}^{\frac{\pi}{2}} \frac{|\sin (2 n+1) t|}{\sin t} d t=\frac{2}{\pi} \sum_{k=0}^{2 n} \int_{t_{k}}^{t_{k+1}} \frac{|\sin (2 n+1) t|}{\sin t} d t
$$




$$
\begin{aligned}
= & \frac{2}{\pi} \int_{t_{0}}^{t_{1}} \frac{|\sin (2 n+1) t|}{\sin t} d t+\frac{2}{\pi} \sum_{k=1}^{n}\left(\int_{t_{2 k-1}}^{t_{2 k}} \frac{|\sin (2 n+1) t|}{\sin t} d t+\int_{t_{2 k}}^{t_{2 k+1}} \frac{|\sin (2 n+1) t|}{\sin t} d t\right) \\
= & \frac{2}{\pi} \int_{0}^{T} \frac{|\sin (2 n+1) t|}{\sin t} d t \\
& +\frac{2}{\pi} \sum_{k=1}^{n}\left(\int_{0}^{T} \frac{\left|\sin (2 n+1)\left(u+t_{2 k-1}\right)\right|}{\sin \left(u+t_{2 k-1}\right)} d u+\int_{0}^{T} \frac{\left|\sin (2 n+1)\left(v+t_{2 k}\right)\right|}{\sin \left(v+t_{2 k}\right)} d v\right) \\
= & \frac{2}{\pi} \int_{0}^{T} \frac{|\sin (2 n+1) t|}{\sin t} d t \\
& +\frac{2}{\pi} \sum_{k=1}^{n}\left(\int_{0}^{T} \frac{\left|\sin \left(\frac{\pi(2 k-1)}{2}+(2 n+1) u\right)\right|}{\sin \left(u+t_{2 k-1}\right)} d u+\int_{0}^{T} \frac{|\sin (\pi k+(2 n+1) v)|}{\sin \left(v+t_{2 k}\right)} d v\right) \\
= & \frac{2}{\pi} \int_{0}^{T} \frac{|\sin (2 n+1) t|}{\sin t} d t+\frac{2}{\pi} \sum_{k=1}^{n}\left(\int_{0}^{T} \frac{|\cos (2 n+1) u|}{\sin \left(u+t_{2 k-1}\right)} d u+\int_{0}^{T} \frac{|\sin (2 n+1) v|}{\sin \left(v+t_{2 k}\right)} d v\right) \\
= & \frac{2}{\pi} \int_{0}^{T} \frac{\sin (2 n+1) t}{\sin t} d t+\frac{2}{\pi} \sum_{k=1}^{n} \int_{0}^{T}\left(\frac{\cos (2 n+1) t}{\sin \left(t+t_{2 k-1}\right)} d t+\frac{\sin (2 n+1) t}{\sin \left(t+t_{2 k}\right)} d t\right. \\
= & I_{0}(n)+I(n),
\end{aligned}
$$

where $T=\frac{\pi}{4 n+2}, n \in \mathbb{N}$. The proof is complete.

Theorem 2. Term (24) of Lebesgue constant (23) satisfies the relations

$$
\theta<I_{0}(n)<\varphi_{1}(n), \quad n \in \mathbb{N}, \quad \varphi_{1}(n) \equiv \frac{\pi}{2} \theta \alpha_{n},
$$

where the function $\alpha_{n}$ is defined in (9),

$$
\theta=\sum_{k=1}^{+\infty} \frac{(-1)^{k}}{(2 k+1)(2 k+1) !}\left(\frac{\pi}{2}\right)^{2 k}=0.872654299 \ldots
$$

Proof. In domain $(0, T]$ we consider the following equivalent two-sided estimates:

$$
\begin{aligned}
\frac{\sin \frac{\pi}{4 n+2}}{\frac{\pi}{4 n+2}} t & \leqslant \sin t<t \Leftrightarrow \frac{1}{t}<\frac{1}{\sin t} \leqslant \frac{\frac{\pi}{4 n+2}}{\sin \frac{\pi}{4 n+2}} \frac{1}{t} \\
& \Leftrightarrow \frac{\sin (2 n+1) t}{t}<\frac{\sin (2 n+1) t}{\sin t} \leqslant \frac{\frac{\pi}{4 n+2}}{\sin \frac{\pi}{4 n+2}} \frac{\sin (2 n+1) t}{t}, \quad t \in\left(0, \frac{\pi}{4 n+2}\right] .
\end{aligned}
$$

At the point $t=0$, we define the functions $y=\frac{\sin (2 n+1) t}{\sin t}, y=\frac{\sin (2 n+1) t}{t}$ via its right-sided limits $y(0)=y(+0)=2 n+1$. Then we multiply two-sided inequality (29) by the constant $\frac{2}{\pi}$ and we integrate then over the domain $[0, T]$. As a result, we obtain strict inequalities of form:

$$
\frac{2}{\pi} \int_{0}^{T} \frac{\sin (2 n+1) t}{t} d t<\frac{2}{\pi} \int_{0}^{T} \frac{\sin (2 n+1) t}{\sin t} d t<\frac{\frac{\pi}{4 n+2}}{\sin \frac{\pi}{4 n+2}} \frac{2}{\pi} \int_{0}^{T} \frac{\sin (2 n+1) t}{t} d t
$$




$$
\Leftrightarrow \frac{2}{\pi} \int_{0}^{T} \frac{\sin (2 n+1) t}{t} d t<I_{0}(n)<\frac{\pi}{2} \alpha_{n} \frac{2}{\pi} \int_{0}^{T} \frac{\sin (2 n+1) t}{t} d t .
$$

We express the integral involved in the last inequality via the series:

$$
\begin{aligned}
& \frac{2}{\pi} \int_{0}^{T} \frac{\sin (2 n+1) t}{t} d t \\
& \quad=\frac{2}{\pi} \int_{0}^{T}\left((2 n+1)-\frac{1}{3 !}(2 n+1)^{3} t^{2}+\frac{1}{5 !}(2 n+1)^{5} t^{4}-\frac{1}{7 !}(2 n+1)^{7} t^{6}+\ldots\right) d t \\
& \quad=\left.\frac{2}{\pi}\left((2 n+1) t-\frac{1}{3 \cdot 3 !}(2 n+1)^{3} t^{3}+\frac{1}{5 \cdot 5 !}(2 n+1)^{5} t^{5}-\frac{1}{7 \cdot 7 !}(2 n+1)^{7} t^{7}+\ldots\right)\right|_{0} ^{T} \\
& \quad=1-\frac{1}{3 \cdot 3 !}\left(\frac{\pi}{2}\right)^{2}+\frac{1}{5 \cdot 5 !}\left(\frac{\pi}{2}\right)^{4}-\frac{1}{7 \cdot 7 !}\left(\frac{\pi}{2}\right)^{6}+\ldots=\sum_{k=0}^{+\infty} \frac{(-1)^{k}}{(2 k+1)(2 k+1) !}\left(\frac{\pi}{2}\right)^{2 k} \equiv \theta .
\end{aligned}
$$

We have obtained a fast convergent sign-changing series, whose sum coincides with the value $\frac{2}{\pi} \operatorname{Si}\left(\frac{\pi}{2}\right)$ (see formulae (10), (28)). Taking into consideration its first seven terms, we get the two-sided estimate for its sum $0.87265429946<\theta<0.87265429948$. The proof is complete.

Let us introduce the notations allowing us to write compactly the formulae for $L_{n}$ obtained on the base of Theorem 1:

$$
\begin{aligned}
& y_{2 k-1}=y\left(t_{2 k-1}\right)=\frac{1}{\sin t_{2 k-1}}, \quad y_{2 k}=y\left(t_{2 k}\right)=\frac{1}{\sin t_{2 k}}, \quad k=1, \ldots, n, \\
& S(n)=\frac{4}{\pi(2 n+1)} \sum_{k=1}^{n} y_{2 k}=\frac{4}{\pi(2 n+1)} \sum_{k=1}^{n} \frac{1}{\sin t_{2 k}}, \\
& I_{1}(n)=\frac{2}{\pi(2 n+1)} \sum_{k=1}^{n} \int_{0}^{T}\left(\frac{\sin (2 n+1) t \cos \left(t+t_{2 k-1}\right)}{\sin ^{2}\left(t+t_{2 k-1}\right)}-\frac{\cos (2 n+1) t \cos \left(t+t_{2 k}\right)}{\sin ^{2}\left(t+t_{2 k}\right)}\right) d t, \\
& I_{2}(n)=\frac{2}{\pi(2 n+1)^{2}} \sum_{k=1}^{n} \int_{0}^{T}\left(\frac{\cos (2 n+1) t\left(1+\cos ^{2}\left(t+t_{2 k-1}\right)\right)}{\sin ^{3}\left(t+t_{2 k-1}\right)}\right. \\
& \left.\quad+\frac{\sin (2 n+1) t\left(1+\cos ^{2}\left(t+t_{2 k}\right)\right)}{\sin ^{3}\left(t+t_{2 k}\right)}\right) d t,
\end{aligned}
$$

where $n \in \mathbb{N}$ and the nodes $t_{j}$ were defined in $(26)$.

Theorem 3. Second term (25) of constant (23) satisfies the following representations:

$$
\begin{aligned}
& I(n)=S(n)+I_{1}(n), \quad n \in \mathbb{N}, \\
& I(n)=S(n)+\varphi_{3}(n)-I_{2}(n), \quad n \in \mathbb{N},
\end{aligned}
$$

where the functions in the right hand sides were defined respectively in (31), (32), (17) and (33).

Proof. We integrate by parts in the integrals in sum (25). In the first group of the integral we make the changes

$$
u=\frac{1}{\sin \left(t+t_{2 k-1}\right)}, \quad d v=\cos (2 n+1) t d t, \quad k=1, \ldots, n, \quad t_{2 k-1}=\frac{(2 k-1) \pi}{4 n+2},
$$


while the changes for the second group are

$$
u=\frac{1}{\sin \left(t+t_{2 k}\right)}, \quad d v=\sin (2 n+1) t d t, \quad k=1, \ldots, n, \quad t_{2 k}=\frac{2 k \pi}{4 n+2} .
$$

After some transformations we obtain

$$
\begin{aligned}
I(n)= & \frac{2}{\pi} \sum_{k=1}^{n} \int_{0}^{T}\left(\frac{\cos (2 n+1) t}{\sin \left(t+t_{2 k-1}\right)} d t+\frac{\sin (2 n+1) t}{\sin \left(t+t_{2 k}\right)}\right) d t \\
= & \frac{2}{\pi} \sum_{k=1}^{n}\left(\left.\frac{\sin (2 n+1) t}{2 n+1} \frac{1}{\sin \left(t+t_{2 k-1}\right)}\right|_{0} ^{T}+\int_{0}^{T} \frac{\sin (2 n+1) t \cos \left(t+t_{2 k-1}\right)}{(2 n+1) \sin ^{2}\left(t+t_{2 k-1}\right)} d t\right. \\
& \left.+\left.\left(-\frac{\cos (2 n+1) t}{2 n+1} \frac{1}{\sin \left(t+t_{2 k}\right)}\right)\right|_{0} ^{T}-\int_{0}^{T} \frac{\cos (2 n+1) t \cos \left(t+t_{2 k}\right)}{(2 n+1) \sin ^{2}\left(t+t_{2 k}\right)} d t\right) \\
= & \frac{2}{\pi} \sum_{k=1}^{n}\left(\frac{2}{2 n+1} \frac{1}{\sin t_{2 k}}\right. \\
= & \left.\frac{1}{\pi(2 n+1)} \sum_{k=1}^{T} y_{2 k}\left(\frac{\sin (2 n+1) t \cos \left(t+t_{2 k-1}\right)}{\sin ^{2}\left(t+t_{2 k-1}\right)}-\frac{\cos (2 n+1) t \cos \left(t+t_{2 k}\right)}{\sin ^{2}\left(t+t_{2 k}\right)}\right) d t\right) \\
& +\frac{2}{\pi(2 n+1)} \sum_{k=1}^{n} \int_{0}^{T}\left(\frac{\sin (2 n+1) t \cos \left(t+t_{2 k-1}\right)}{\sin ^{2}\left(t+t_{2 k-1}\right)}-\frac{\cos (2 n+1) t \cos \left(t+t_{2 k}\right)}{\sin ^{2}\left(t+t_{2 k}\right)}\right) d t
\end{aligned}
$$

where the values of the functions $y_{j}$ were defined in (30). Employing notations (31) and (32) in the obtained identity, we complete the proof of formula (34).

We integrate by parts in (32). Now in the first group of the integrals in $I_{1}(n)$ we make the changes

$$
u=\frac{\cos \left(t+t_{2 k-1}\right)}{\sin ^{2}\left(t+t_{2 k-1}\right)}, \quad d v=\sin (2 n+1) t d t, \quad k=1, \ldots, n,
$$

while in the second group we make the changes

$$
u=\frac{\cos \left(t+t_{2 k}\right)}{\sin ^{2}\left(t+t_{2 k}\right)}, \quad d v=\cos (2 n+1) t d t, \quad k=1, \ldots, n .
$$

Then we simplify them:

$$
\begin{aligned}
I_{1}(n)=\frac{2}{\pi(2 n+1)} \sum_{k=1}^{n}\left(-\left.\frac{\cos (2 n+1) t}{2 n+1} \frac{\cos \left(t+t_{2 k-1}\right)}{\sin ^{2}\left(t+t_{2 k-1}\right)}\right|_{0} ^{T}-\int_{0}^{T} \frac{\cos (2 n+1) t\left(1+\cos ^{2}\left(t+t_{2 k-1}\right)\right)}{(2 n+1) \sin ^{3}\left(t+t_{2 k-1}\right)} d t\right. \\
\left.\quad-\left.\frac{\sin (2 n+1) t}{2 n+1} \frac{\cos \left(t+t_{2 k}\right)}{\sin ^{2}\left(t+t_{2 k}\right)}\right|_{0} ^{T}-\int_{0}^{T} \frac{\sin (2 n+1) t\left(1+\cos ^{2}\left(t+t_{2 k}\right)\right)}{(2 n+1) \sin ^{3}\left(t+t_{2 k}\right)} d t\right) \\
=\frac{2}{\pi(2 n+1)^{2}} \sum_{k=1}^{n}\left(\frac{\cos t_{2 k-1}}{\sin ^{2} t_{2 k-1}}-\frac{\cos t_{2 k+1}}{\sin ^{2} t_{2 k+1}}\right)
\end{aligned}
$$




$$
\begin{gathered}
-\frac{2}{\pi(2 n+1)^{2}} \sum_{k=1}^{n} \int_{0}^{T}\left(\frac{\cos (2 n+1) t\left(1+\cos ^{2}\left(t+t_{2 k-1}\right)\right)}{\sin ^{3}\left(t+t_{2 k-1}\right)}\right. \\
\left.+\frac{\sin (2 n+1) t\left(1+\cos ^{2}\left(t+t_{2 k}\right)\right)}{\sin ^{3}\left(t+t_{2 k}\right)}\right) d t \\
=\frac{2}{\pi(2 n+1)^{2}}\left(\frac{\cos t_{1}}{\sin ^{2} t_{1}}-\frac{\cos t_{2 n+1}}{\sin ^{2} t_{2 n+1}}\right)-I_{2}(n) \\
=\frac{2 \cos t_{1}}{\pi\left((2 n+1) \sin t_{1}\right)^{2}}-I_{2}(n)=\frac{2}{\pi} \alpha_{n}^{2} \cos \frac{\pi}{4 n+2}-I_{2}(n) .
\end{gathered}
$$

In view of notation (17), we rewrite the obtained identity as

$$
I_{1}(n)=\varphi_{3}(n)-I_{2}(n), \quad n \in \mathbb{N} .
$$

This allows to obtain formula (35) from formula (34). The proof is complete.

Remark 3. The Lebesgue constant satisfy the representations

$$
\begin{aligned}
& L_{n}=I_{0}(n)+S(n)+I_{1}(n), \quad n \in \mathbb{N}, \\
& L_{n}=I_{0}(n)+S(n)+\varphi_{3}(n)-I_{2}(n), \quad n \in \mathbb{N},
\end{aligned}
$$

which are simple corollaries of Theorems 1 and 3 (see formulae (23), (34), (35)). In the next section these representations will allow us to get strict upper and lower bound for the constant $L_{n}$.

\section{TWO-SIDED ESTimates FOR LEBESGUE CONSTANT}

We shall employ several first exact values of the Lebesgue constants $L_{n}=L(n), n \in \mathbb{N}$, $L(0)=1$ in the proofs of theorems on upper bound of $L_{n}$. Let us calculate them according formulae (23)-(25) or (5):

$$
\begin{aligned}
& L(1)=\frac{1}{3}+\frac{2 \sqrt{3}}{\pi}=1.435991124 \ldots, \\
& L(2)=\frac{1}{5}+\frac{2}{\pi}\left(-\sin \frac{\pi}{5}+3 \sin \frac{2 \pi}{5}\right)=1.642188435 \ldots, \\
& L(3)=\frac{1}{7}+\frac{2}{3 \pi}\left(11 \sin \frac{\pi}{7}+5 \sin \frac{2 \pi}{7}-\sin \frac{3 \pi}{7}\right)=1.778322861 \ldots
\end{aligned}
$$

We observe the complexity of the calculations grows fast as $n$ increases. Therefore, from the practical point of view, it is important to find a good approximation formula of form

$$
L_{n} \approx \frac{4}{\pi^{2}} \ln n+A, \quad n \in \mathbb{N}, \quad A \in[1,3] \subset(0, \infty)
$$

for calculating the values of the constants $L_{n}, n \geqslant 1$. We observe that reducing of the domain of the parameter $A$ in (40) to the segment $[1,3]$ is in agreement with the known results mentioned in the introduction.

In this section we improve the known lower and upper bounds for the Lebesgue constants $L_{n}$.

Problem 1. Find a monotonically decreasing sequence $A_{1}>A_{2}>A_{3}>\ldots, A_{k} \in[1,3]$, whose elements satisfy the inequalities

$$
L_{n} \leqslant A_{k}+\frac{4}{\pi^{2}} \ln n \equiv \mu_{k}(n), \quad n \in \mathbb{N}, \quad k=1,2,3, \ldots
$$

uniformly in the parameter $n$. 
Employing the results and the notations of the previous section, we first prove a theorem on a two-sided estimate for constant (3).

Theorem 4. Lebesgue constant (3) satisfy the two-sided inequality

$$
I_{0}(n)+S(n)<L_{n}<I_{0}(n)+S(n)+\varphi_{3}(n), \quad n \in \mathbb{N},
$$

with the terms defined by formulae (24), (31) and (17).

Proof. 1. In order to prove the upper bound in (42), we employ formula (38). According the latter, it is sufficient to establish that the sum $I_{2}(n)$ (see (33)) is positive for all natural $n$. Indeed, all integrands in formula (33) are positive and the same is true for their integrals over the domain $[0, T]$. Therefore,

$$
I_{2}(n)>0, \quad n \in \mathbb{N} \Rightarrow L_{n}<I_{0}(n)+S(n)+\varphi_{3}(n), \quad n \in \mathbb{N} .
$$

2. In order to prove the lower bound in (42), it is sufficient to establish the positivity of the last term in formula (37), that is, to prove the inequality $I_{1}(n)>0, n \in \mathbb{N}$. Employing formula (32) and notations

$$
\begin{aligned}
& y=g_{n}(t)=\sum_{k=1}^{n} \frac{\cos \left(t+t_{2 k-1}\right)}{\sin ^{2}\left(t+t_{2 k-1}\right)}=\sum_{k=1}^{n} \operatorname{cosec}\left(t+t_{2 k-1}\right) \cot \left(t+t_{2 k-1}\right), \quad t \in[0, T], \\
& y=h_{n}(t)=\sum_{k=1}^{n} \frac{\cos \left(t+t_{2 k}\right)}{\sin ^{2}\left(t+t_{2 k}\right)}=\sum_{k=1}^{n} \operatorname{cosec}\left(t+t_{2 k}\right) \cot \left(t+t_{2 k}\right), \quad t \in[0, T], \quad n \in \mathbb{N},
\end{aligned}
$$

we rewrite the inequality $I_{1}(n)>0$ in the equivalent form

$$
\bar{I}_{1}(n)>0, \quad n \in \mathbb{N}, \quad \bar{I}_{1}(n)=\int_{0}^{T}\left(g_{n}(t) \sin (2 n+1) t-h_{n}(t) \cos (2 n+1) t\right) d t .
$$

We first study the behavior of the functions (43), (44) in the Cartesian coordinates $t O y$. They are positive, concave and decrease in their domains. As one can confirm easily, this is true for cosecants, cotangents and their products in the corresponding subdomains of the domain $\left[T, \frac{\pi}{2}\right]$. These functions and their derivatives satisfy the relations

$$
\begin{aligned}
& g_{n}(t)>h_{n}(t), \quad t \in[0, T], \quad n \in \mathbb{N}, \\
& g_{n}^{\prime}(t)<h_{n}^{\prime}(t)<0\left(\Leftrightarrow\left|g_{n}^{\prime}(t)\right|>\left|h_{n}^{\prime}(t)\right|\right), \quad g_{n}^{\prime \prime}(t)>h_{n}^{\prime \prime}(t)>0, \quad t \in[0, T], \quad n \in \mathbb{N} .
\end{aligned}
$$

Inequality (46) follows immediately by the definitions of functions (43), (44) and their properties: for each value of the independent variable $t$ and the parameter $n$, each term $\operatorname{cosec}(t+$ $\left.t_{2 k-1}\right) \cot \left(t+t_{2 k-1}\right)$ in sum (43) is greater than the corresponding term $\operatorname{cosec}\left(t+t_{2 k}\right) \cot \left(t+t_{2 k}\right)$ in (44). Inequalities (47) can be proved by applying the same arguing to the derivatives explicitly written in terms of cotangents and cosecants. In short, these inequalities obviously express the geometric properties of the functions $g_{n}, h_{n}$.

We shall make of the equations of straight lines (subtenses $y=L_{n}(t), y=l_{n}(t)$ ) passing though the known boundary points $A_{1}\left(0, g_{n}(0)\right), A_{2}\left(T, g_{n}(T)\right)$ and $B_{1}\left(0, h_{n}(0)\right), B_{2}\left(T, h_{n}(T)\right)$ of the graphs of the functions (43) and (44), respectively:

$$
\begin{aligned}
& L_{n}(t)=a_{1}+k_{1} t, \quad a_{1}=a_{1}(n)=g_{n}(0), \quad k_{1}=k_{1}(n)=\frac{g_{n}(T)-g_{n}(0)}{T}, \quad t \in[0, T], \\
& l_{n}(t)=a_{2}+k_{2} t, \quad a_{2}=a_{2}(n)=h_{n}(0), \quad k_{2}=k_{2}(n)=\frac{h_{n}(T)-h_{n}(0)}{T}, \quad n \in \mathbb{N},
\end{aligned}
$$


where the coefficients of the straight lines (48), (49) were expressed via certain quantities:

$$
g_{n}(0)=\sum_{k=1}^{n} \frac{\cos t_{2 k-1}}{\sin ^{2} t_{2 k-1}}, \quad g_{n}(T)=h_{n}(0)=\sum_{k=1}^{n} \frac{\cos t_{2 k}}{\sin ^{2} t_{2 k}}, \quad h_{n}(T)=\sum_{k=1}^{n} \frac{\cos t_{2 k+1}}{\sin ^{2} t_{2 k+1}} .
$$

Since the functions $g_{n}(t), h_{n}(t), n \in \mathbb{N}$, are concave and decrease in the domain $[0, T]$ and their subtenses satisfy relations (46)-(50), we have

$$
\begin{aligned}
& g_{n}(t)<L_{n}(t), \quad h_{n}(t)<l_{n}(t), \quad t \in(0, T), \\
& g_{n}(0)=L_{n}(0), \quad g_{n}(T)=L_{n}(T)=l_{n}(0), \quad h_{n}(T)=l_{n}(T), \\
& L_{n}(t)-g_{n}(t) \geqslant l_{n}(t)-h_{n}(t) \geqslant 0, \quad t \in[0, T],
\end{aligned}
$$

and the identity in (52) is attained only at the end-points of the considered domain.

As an implication of relations (49)-(51), we obtain the chain of inequalities

$$
g_{n}(t)>g_{n}(T)>l_{n}(t)>h_{n}(t)>0, \quad t \in(0, T), \quad n \in \mathbb{N},
$$

which will be used in proving the lower bound for the Lebesgue constant. We employ inequalities (53) and the properties of the Riemann integral to estimate from below the discrete function $\bar{I}_{1}(n)$. In order to do this, we decrease the first integrand in (45) and increase the second. As a result we obtain

$$
\begin{aligned}
\bar{I}_{1}(n) & >\int_{0}^{T} g_{n}(T) \sin (2 n+1) t d t-\int_{0}^{T} l_{n}(t) \cos (2 n+1) t d t \\
& =g_{n}(T) \int_{0}^{T} \sin (2 n+1) t d t-\int_{0}^{T} \cos (2 n+1) t\left(a_{2}+k_{2} t\right) d t \\
& =\frac{g_{n}(T)}{2 n+1}-\left(\left.\frac{a_{2}+k_{2} t}{2 n+1} \sin (2 n+1) t\right|_{0} ^{T}-\int_{0}^{T} \frac{k_{2} \sin (2 n+1) t}{2 n+1} d t\right) \\
& =\frac{h_{n}(0)}{2 n+1}-\left(\frac{a_{2}}{2 n+1}+k_{2}\left(\frac{\pi}{2(2 n+1)^{2}}-\frac{1}{(2 n+1)^{2}}\right)\right) \\
& =\frac{h_{n}(0)}{2 n+1}-\left(\frac{h_{n}(0)}{2 n+1}+\frac{\pi-2}{2(2 n+1)^{2}} \frac{h_{n}(T)-h_{n}(0)}{T}\right) \\
& =\frac{\pi-2}{\pi(2 n+1)}\left(h_{n}(0)-h_{n}(T)\right)>0, \quad n \in \mathbb{N} .
\end{aligned}
$$

In addition, we have employed here the relations $g_{n}(T)=h_{n}(0), h_{n}(0)>h_{n}(T)$.

The established inequality, the equivalence

$$
\bar{I}_{1}(n)>0, \quad n \in \mathbb{N} \quad \Leftrightarrow \quad I_{1}(n)>0, \quad n \in \mathbb{N},
$$

and formula (37) allow us to prove the lower bound in (42). The proof is complete.

As we see in the proof of Theorem 4, the Lebesgue constant was estimated from above and below rather rough. Despite of this, main inequality (42) will allow us to obtain sharp inequalities for constant (3). In literature, the lower bounds for $L_{n}$ were studied more rarely than the upper ones. A similar situation holds in the case of the Lagrange interpolation [24]. The said is related to specific problems arising in proving these bounds. However, the results of Theorems 2 and 4 as well as Lemma 4 will allows us to estimate rather sharply the constant $L_{n}$ from below. 
Theorem 5. Lebesgue constant (3) satisfies the uniform lower bound

$$
L_{n}>1.173198+\frac{4}{\pi^{2}} \ln n, \quad n \in \mathbb{N} .
$$

Proof. According inequality (42), to prove the theorem, we need to study in details the behavior of the integral $I_{0}(n)$ and of the sum $S(n)$. The study of the former was done in Theorem 2 . The sum $S(n)$ is involved in both the upper and lower estimate of main inequality (42) and this is why we shall estimate this sum both from above and below. In order to do this, we rewrite (31) in a form more convenient for further study:

$$
S(n)=\frac{4}{\pi^{2}} \sum_{k=1}^{n} y_{2 k} \frac{\pi}{2 n+1}=\frac{4}{\pi^{2}} \sum_{k=1}^{n} \frac{1}{\sin \frac{\pi k}{2 n+1}} \frac{\pi}{2 n+1}, \quad n \in \mathbb{N} .
$$

It is clear that sum (55) is the approximate formula of mean rectangles over the uniformly distributed in the segment $\left[T, \frac{\pi}{2}\right]$ nodes for the integral

$$
i(n)=\frac{4}{\pi^{2}} \int_{T}^{\frac{\pi}{2}} \frac{d t}{\sin t}, \quad T=\frac{\pi}{4 n+2} .
$$

In our case, that is, for the concave and decreasing in the bounded domain $\left[T, \frac{\pi}{2}\right]$ function $y=\frac{1}{\sin t}$, sum (55) satisfies the two-sided estimate

$$
\frac{4}{\pi^{2}} \frac{\pi}{2(2 n+1)} \frac{1}{\sin \frac{\pi}{2 n+1}}+\frac{4}{\pi^{2}} \int_{t_{2}}^{\frac{\pi}{2}} \frac{d t}{\sin t}<S(n)<i(n), \quad n \in \mathbb{N} .
$$

Let us simplify the terms in two-sided inequality (56) by employing Lemmata 2 and 4:

$$
\begin{gathered}
\frac{4}{\pi^{2}} \frac{\pi}{2(2 n+1)} \frac{1}{\sin \frac{\pi}{2 n+1}}=\frac{1}{\pi(2 n+1) \sin \frac{\pi}{4 n+2} \cos \frac{\pi}{4 n+2}}=\frac{\alpha_{n}}{\pi} \sec \frac{\pi}{4 n+2} \equiv \varphi_{6}(n), \\
\frac{4}{\pi^{2}} \int_{t_{2}}^{\frac{\pi}{2}} \frac{d t}{\sin t}=\left.\frac{4}{\pi^{2}} \ln \tan \frac{t}{2}\right|_{\frac{\pi}{2 n+1}} ^{\frac{\pi}{2}}=\frac{4}{\pi^{2}} \ln \frac{\cos \frac{\pi}{4 n+2}}{\sin \frac{\pi}{4 n+2}}=\frac{4}{\pi^{2}} \ln \frac{\cos \frac{\pi}{4 n+2} n(2 n+1)}{\sin \frac{\pi}{4 n+2} n(2 n+1)} \\
=\frac{4}{\pi^{2}} \ln n+\frac{4}{\pi^{2}} \ln \left(\left(2+\frac{1}{n}\right) \alpha_{n} \cos \frac{\pi}{4 n+2}\right) \equiv \frac{4}{\pi^{2}} \ln n+\varphi_{5}(n), \\
i(n)=\frac{4}{\pi^{2}} \int_{T}^{\frac{\pi}{2}} \frac{d t}{\sin t}=\left.\frac{4}{\pi^{2}} \ln \tan \frac{t}{2}\right|_{\frac{\pi}{4 n+2}} ^{\frac{\pi}{2}}=\frac{4}{\pi^{2}} \ln \frac{\cos \frac{\pi}{8 n+4}}{\sin \frac{\pi}{8 n+4}}=\frac{4}{\pi^{2}} \ln \frac{\cos \frac{\pi}{8 n+4} \cdot 2 \cos \frac{\pi}{8 n+4} \cdot n(2 n+1)}{\sin \frac{\pi}{8 n+4} \cdot 2 \cos \frac{\pi}{8 n+4} \cdot n(2 n+1)} \\
=\frac{4}{\pi^{2}} \ln n+\frac{4}{\pi^{2}} \ln \left(\left(2+\frac{1}{n}\right) \alpha_{n}\left(1+\cos \frac{\pi}{4 n+2}\right)\right) \equiv \frac{4}{\pi^{2}} \ln n+\varphi_{2}(n) .
\end{gathered}
$$

These calculations allow us to rewrite inequality (56) in the form

$$
\varphi_{5}(n)+\varphi_{6}(n)+\frac{4}{\pi^{2}} \ln n<S(n)<\varphi_{2}(n)+\frac{4}{\pi^{2}} \ln n, \quad n \in \mathbb{N} .
$$

Now, in view of inequalities (42), (27), (57) and Lemma 4, it is easy to prove inequality (54):

$$
\begin{aligned}
L(n)> & I_{0}(n)+S(n)>\theta+\varphi_{5}(n)+\varphi_{6}(n)+\frac{4}{\pi^{2}} \ln n>\theta+\inf _{n \in \mathbb{N}} \varphi_{5}(n)+\inf _{n \in \mathbb{N}} \varphi_{6}(n)+\frac{4}{\pi^{2}} \ln n \\
& >0.872654+0.0907902+0.202642+\frac{4}{\pi^{2}} \ln n=1.173198+\frac{4}{\pi^{2}} \ln n .
\end{aligned}
$$

The proof is complete. 
In the next two theorems are define specific values of first two constants $A_{1}$ and $A_{2}$ in Problem 1 devoted to the upper bound of the Lebesgue constant.

Theorem 6. The constant $A_{1}=1.705598$ solves Problem 1, that is, the strict inequality

$$
L_{n}<1.705598+\frac{4}{\pi^{2}} \ln n \equiv \tilde{\mu}_{1}(n), \quad n \in \mathbb{N},
$$

holds.

Proof. We estimate Lebesgue constant (3) from above taking into consideration inequalities (42), (27), (57):

$$
L_{n}<I_{0}(n)+S(n)+\varphi_{3}(n)<\varphi_{1}(n)+\varphi_{2}(n)+\varphi_{3}(n)+\frac{4}{\pi^{2}} \ln n, \quad n \in \mathbb{N} .
$$

Employing relations (12), (15), (19) for the functions $\varphi_{1}, \varphi_{2} \in V_{\delta}^{-}, \varphi_{3} \in V_{\delta}^{+}$, we continue the uniform upper bound estimate in (59):

$$
\begin{aligned}
L_{n} & <\max _{n \in \mathbb{N}} \varphi_{1}(n)+\max _{n \in \mathbb{N}} \varphi_{2}(n)+\max _{n \in \mathbb{N}} \varphi_{3}(n)+\frac{4}{\pi^{2}} \ln n \\
& <0.913842+0.533743+0.258013+\frac{4}{\pi^{2}} \ln n=1.705598+\frac{4}{\pi^{2}} \ln n, \quad n \in D_{1}=\mathbb{N} .
\end{aligned}
$$

The proof is complete.

Let us check inequality (58) for the first values of the parameter $n$ by employing exact values of the Lebesgue constant in (39) and let us calculate the made error $\tilde{\varepsilon}_{n}=\tilde{\varepsilon}(n) \equiv \tilde{\mu}_{1}(n)-L(n)$ :

$$
\begin{array}{ll}
n=1: & L(1)=\frac{1}{3}+\frac{2 \sqrt{3}}{\pi}=1.435991 \ldots, \quad \tilde{\mu}_{1}(1)=1.705598+\frac{4}{\pi^{2}} \ln 1=1.705598 \\
& \Rightarrow L(1)<\tilde{\mu}_{1}(1), \quad \tilde{\varepsilon}_{1} \approx 0.269607 ; \\
n=2: \quad L(2)=\frac{1}{5}+\frac{2}{\pi}\left(3 \sin \frac{2 \pi}{5}-\sin \frac{\pi}{5}\right)=1.642188 \ldots, & \\
& \tilde{\mu}_{1}(2)=1.705598+\frac{4}{\pi^{2}} \ln 2 \approx 1.986919 \Rightarrow L(2)<\tilde{\mu}_{1}(2), \quad \tilde{\varepsilon}_{2} \approx 0.344731 ; \\
n=3: \quad L(3)=\frac{1}{7}+\frac{2}{\pi}\left(-\frac{1}{3} \sin \frac{4 \pi}{7}+\frac{5}{3} \sin \frac{2 \pi}{7}+\frac{11}{3} \sin \frac{\pi}{7}\right)=1.778322 \ldots,
\end{array}
$$

The inequalities hold, their left hand sides and right hand sides differ by a small quantity. As the parameter $n$ grows, we see that the error $\tilde{\varepsilon}_{n}$ increase and then $(n \gg 1)$, it will stabilizes at certain number.

The solution $A_{1}=1.705598$ to Problem 1 allows us to state that there exists many other its solutions satisfying the inequalities $A_{1}>A_{2}>A_{3}>\ldots>A_{k}>\ldots$ In what follows we find a specific value $A_{2}$ employing Lemmata 1-3 established for the domain $D_{2}=\{3,4,5,6, \ldots\} \subset \mathbb{N}$.

Theorem 7. The constant $A_{2}=1.577629, A_{2}<A_{1}$, solves Problem 1, that is, the strict inequality

$$
L_{n}<1.577629+\frac{4}{\pi^{2}} \ln n \equiv \tilde{\mu}_{2}(n), \quad n \in \mathbb{N},
$$

holds.

Proof. Let us first prove estimate (60) in the subdomain $D_{2}$ of the set of natural numbers $\mathbb{N}$. In order to do this, we employ inequality (59) (it holds automatically for all $n$ in the domain $D_{2}$ ), 
in which we estimate the functions $L(n), \varphi_{k}(n), k=1,2,3$, from above employing relations (13), (16), (20):

$$
\begin{aligned}
L_{n} & <\max _{n \in D_{2}} \varphi_{1}(n)+\max _{n \in D_{2}} \varphi_{2}(n)+\max _{n \in D_{2}} \varphi_{3}(n)+\frac{4}{\pi^{2}} \ln n \\
& <0.880022+0.439594+0.2580134+\frac{4}{\pi^{2}} \ln n=1.577629+\frac{4}{\pi^{2}} \ln n=\tilde{\mu}_{2}(n), \quad n \in D_{2} .
\end{aligned}
$$

Thus, inequality (41) holds for the constant $A_{2}=1.577629, A_{2}<A_{1}$, in the subdomain $D_{2} \subset \mathbb{N}$.

Let us check the obtained inequality for the values $n=1$ and $n=2$ not involved in the domain $D_{2}$ :

$$
\begin{aligned}
& n=1: \quad L(1)=1.435991 \ldots, \quad \tilde{\mu}_{2}(1)=1.577629+\frac{4}{\pi^{2}} \ln 1=1.577629 \Rightarrow L(1)<\tilde{\mu}_{2}(1), \\
& n=2: \quad L(2)=1.642188 \ldots, \quad \tilde{\mu}_{2}(2)=1.577629+\frac{4}{\pi^{2}} \ln 2 \approx 1.858551 \Rightarrow L(2)<\tilde{\mu}_{2}(2) .
\end{aligned}
$$

These inequalities hold and this proves inequality (60). The proof is complete.

Remark 4. The error term $O_{n} \equiv L_{n}-\frac{4}{\pi^{2}} \ln n$ of the Lebesgue function satisfies the uniform two-sided estimate

$$
1.173198<L_{n}-\frac{4}{\pi^{2}} \ln n<1.577629, \quad n \in \mathbb{N},
$$

which is a direct corollary of Theorem 5 and 7 .

Remark 5. Seeking the sequence $A_{k}$ in Problem 1 by means of Theorem 4 does not lead us to finding the extremal value $A^{*}$ in (8). This is implied from the statements of Lemmata 1-3 on the ranges $R\left(\varphi_{k}\right)$ of functions $\varphi_{k}=\varphi_{k}(n), k=1,2,3$. This is why we shall solve problem (8) in another way.

\section{EXTREMAL PROBLEM RELATED WITH UPPER BOUND OF $L_{n}$}

Employing the notations of the previous section, we formulate problem (8) in a form more convenient for further studying.

Problem 2. Among all solutions to Problem 1, find the smallest constant $A^{*}=\min _{k \in \mathbb{N}} A_{k}$, for which the inequality

holds uniformly in the parameter $n$.

$$
L_{n} \leqslant A^{*}+\frac{4}{\pi^{2}} \ln n \equiv \tilde{\mu}(n), \quad n \in \mathbb{N},
$$

We shall solve this extremal problem related to the upper bound for the Lebesgue constant on the base of strengthening Theorem 4, see Remark 5. We note that in the framework of the present work, we do not study the issues related to a lower bound for the constant $L_{n}$ and considered and partially solved in the previous section as well as its extremal version. Solving an analogue of Problem 2 for this case requires additional efforts.

In the Cartesian coordinate system $n O y$, we consider the functions

$$
y=L(n), \quad y=\tilde{\mu}_{1}(n), \quad y=\tilde{\mu}_{2}(n), \quad y=\tilde{\mu}(n), \quad n \in \mathbb{N},
$$

involved in relations (23), (58), (60) and (62), respectively. The first of them (the Lebesgue constant) behaves as the logarithmic function up to some error but it is not the logarithmic function. The other functions in (63) can be obtained one from another by the parallel translation of their graphs along the axis $O n$ keeping at that their vertices at the straight line $n-1=0$.

In view of the above studies of the Lebesgue function and geometric considerations, we can assume that the solution to extremal problem 2 is the constant $A^{*}=L(1)=\frac{1}{3}+\frac{2 \sqrt{3}}{\pi}$. In 
order to justify this conjecture, we return back to identity (37), in which we estimate the term $I_{1}(n)$ (see (32)) in another way. Earlier, in the proof of Theorem 4, we employed the inequality $I_{1}(n)<\varphi_{3}(n), n \in \mathbb{N}$. In order to obtain a sharper upper bound for sum (32), we represent at as the difference of two integrals:

$$
I_{1}(n)=\tilde{I}_{1}(n)-\tilde{I}_{\varepsilon}(n), \quad n \in \mathbb{N},
$$

where

$$
\begin{aligned}
\tilde{I}_{1}(n) & =\frac{2}{\pi(2 n+1)} \int_{0}^{T}\left(L_{n}(t) \sin (2 n+1) t-l_{n}(t) \cos (2 n+1) t\right) d t \\
& =\frac{2}{\pi(2 n+1)} \int_{0}^{T} L_{n}(t) \sin (2 n+1) t d t-\frac{2}{\pi(2 n+1)} \int_{0}^{T} l_{n}(t) \cos (2 n+1) t d t, \\
\tilde{I}_{\varepsilon}(n) & =\frac{2}{\pi(2 n+1)} \int_{0}^{T}\left(\left(L_{n}(t)-g_{n}(t)\right) \sin (2 n+1) t-\left(l_{n}(t)-h_{n}(t)\right) \cos (2 n+1) t\right) d t,
\end{aligned}
$$

the functions $g_{n}(t), h_{n}(t), L_{n}(t), l_{n}(t)$ were defined in (43), (44), (48), (49), respectively.

First we calculate the exact value of the integral $\tilde{I}_{1}(n)$. In order to do this, we integrate by parts in the difference of the integrals in (65) with $u=L_{n}(t), d v=\sin (2 n+1) t d t$ and $u=l_{n}(t), d v=\cos (2 n+1) t d t$. Then we calculate them by employing relations (48), (49):

$$
\begin{aligned}
\tilde{I}_{1}(n) & =\frac{2}{\pi(2 n+1)}\left(\int_{0}^{T}\left(k_{1} t+a_{1}\right) \sin (2 n+1) t d t-\int_{0}^{T}\left(k_{2} t+a_{2}\right) \cos (2 n+1) t d t\right) \\
& =\frac{2}{\pi(2 n+1)}\left(\left.\left(-\frac{k_{1} t+a_{1}}{2 n+1} \cos (2 n+1) t\right)\right|_{0} ^{T}+\frac{k_{1}}{2 n+1} \int_{0}^{T} \cos (2 n+1) t d t\right. \\
& \left.=\left.\frac{2}{\pi(2 n+1)}\left(\frac{k_{1}+k_{2}}{(2 n+1)^{2}}+\frac{a_{1}-a_{2}-k_{2} T}{2 n+1}\right)\right|_{0} ^{T}-\frac{k_{2}}{2 n+1} \sin (2 n+1) t d t\right) \\
& =\frac{2}{\pi(2 n+1)}\left(\frac{1}{(2 n+1)^{2}} \frac{h_{n}(T)-g_{n}(0)}{T}+\frac{1}{(2 n+1)}\left(g_{n}(0)-h_{n}(T)\right)\right) \\
& =\frac{2}{\pi(2 n+1)} \frac{1}{2 n+1}\left(1-\frac{2}{\pi}\right)\left(g_{n}(0)-h_{n}(T)\right) \\
& =\left(\frac{2}{\pi}-\frac{4}{\pi^{2}}\right) \frac{1}{(2 n+1)^{2}} \sum_{k=1}^{n}\left(\frac{\cos _{2 k-1}}{\sin ^{2} t_{2 k-1}}-\frac{\cos t_{2 k+1}}{\sin ^{2} t_{2 k+1}}\right) \\
& =\left(\frac{2}{\pi}-\frac{4}{\pi^{2}}\right) \frac{1}{(2 n+1)^{2}}\left(\frac{\cos t_{1}}{\sin ^{2} t_{1}}-\frac{\cos _{2 n+1}}{\sin ^{2} t_{2 n+1}}\right) \\
& =\frac{2}{\pi}\left(1-\frac{2}{\pi}\right) \frac{1}{(2 n+1)^{2}} \frac{\cos _{\frac{\pi}{4 n+2}}}{\sin ^{2} \frac{\pi}{4 n+2}} \\
& =\frac{2}{\pi}\left(1-\frac{2}{\pi}\right) \alpha_{n}^{2} \cos \frac{\pi}{4 n+2} \cdot
\end{aligned}
$$


The obtained result and notation (18) allow us to write the following explicit formula for integral (65):

$$
\tilde{I}_{1}(n)=\frac{2}{\pi}\left(1-\frac{2}{\pi}\right) \alpha_{n}^{2} \cos \frac{\pi}{4 n+2} \equiv \varphi_{4}(n), \quad n \in \mathbb{N} .
$$

Now let us study the behavior of the integral $\tilde{I}_{\varepsilon}(n)$. Here we study in details the integrand (error function)

$$
\begin{gathered}
\varepsilon_{n}(t) \equiv p_{n}(t)-q_{n}(t), \\
p_{n}(t) \equiv\left(L_{n}(t)-g_{n}(t)\right) \sin (2 n+1) t, \quad q_{n}(t) \equiv\left(l_{n}(t)-h_{n}(t)\right) \cos (2 n+1) t
\end{gathered}
$$

by means of differential calculus.

1. Function (67) satisfies the relations:

$$
\varepsilon_{n}(0)=\varepsilon_{n}(T)=0, \quad \varepsilon_{n}\left(\frac{T}{2}\right)=\frac{\sqrt{2}}{2}\left[\left(L_{n}\left(\frac{T}{2}\right)-g_{n}\left(\frac{T}{2}\right)\right)-\left(l_{n}\left(\frac{T}{2}\right)-h_{n}\left(\frac{T}{2}\right)\right)\right]>0 .
$$

Moreover, we have $\varepsilon_{n}(t)>0, t \in\left(\frac{T}{2}, T\right)$, since in the considered domain we have the inequalities $\sin (2 n+1) t>\cos (2 n+1) t$ and $L_{n}(t)-g_{n}(t)>l_{n}(t)-h_{n}(t)$ (see $\left.(52)\right)$.

The function $\varepsilon_{n}(t)$ vanishes at one more point $t^{*}=t^{*}(n)$ in the interval $\left(0, \frac{T}{2}\right)$. To justifies this, we consider equivalent equations

$$
\begin{aligned}
& \varepsilon_{n}(t)=0 \Leftrightarrow\left(L_{n}(t)-g_{n}(t)\right) \sin (2 n+1) t=\left(l_{n}(t)-h_{n}(t)\right) \cos (2 n+1) t \\
& \Leftrightarrow \cot (2 n+1) t=\frac{L_{n}(t)-g_{n}(t)}{l_{n}(t)-h_{n}(t)}, \quad t \in(0, T), \quad n \in \mathbb{N},
\end{aligned}
$$

where, according inequality (52), the right hand side of equation (68) is strictly greater than one and therefore, $\cot (2 n+1) t>1, t \in(0, T), n \in \mathbb{N}$. The obtained condition for the right hand side of identity (68) means that the root $t^{*}$ is always located in the domain $\left(0, \frac{T}{2}\right)$.

2. The inequality $\varepsilon_{n}^{\prime}(0)<0$ holds, that is, the error function decreases in some neighbourhood of the zero and is negative. Here

$$
\varepsilon_{n}^{\prime}(0)=h_{n}^{\prime}(0)-l_{n}^{\prime}(0) \equiv \tan \alpha\left(B_{1}\right)-\tan \beta\left(B_{1}\right),
$$

where $\alpha\left(B_{1}\right)$ is the angle between the axis $O t$ and the tangent to the graph of the function $y=h_{n}(t)$ at the point $B_{1}=B_{1}\left(0, h_{n}(0)\right)$, and $\beta\left(B_{1}\right)$ is the angle between the axis $O t$ and the subtense $y=l_{n}(t), t \in[0, T]$. Since $h_{n}(t), t \in[0, T]$, is a concave decreasing function, at the point $B_{1}$ we have $\alpha\left(B_{1}\right)<\beta\left(B_{1}\right)$ (both angles belong to the second quarter). This is why the difference of their tangents is always negative.

At the right end-point of the domain, the inequality $\varepsilon_{n}^{\prime}(T)<0$ holds, where

$$
\varepsilon_{n}^{\prime}(T)=L_{n}^{\prime}(T)-g_{n}^{\prime}(T) \equiv \tan \beta\left(A_{2}\right)-\tan \alpha\left(A_{2}\right),
$$

$\alpha\left(A_{2}\right)$ is the angle between the axis $O t$ and the tangent to the graph of the function $y=g_{n}(t)$ at the point $A_{2}\left(T, g_{n}(T)\right)$, and $\beta\left(A_{2}\right)$ is the angle between the axis $O t$ and the subtense $y=L_{n}(t)$, $t \in[0, T]$, and these angles also belong to the second quarter. Therefore,

$$
\alpha\left(A_{2}\right)>\beta\left(A_{2}\right) \quad \Rightarrow \quad \tan \alpha\left(A_{2}\right)>\tan \beta\left(A_{2}\right) \quad \Rightarrow \quad \varepsilon_{n}^{\prime}(T)<0 .
$$

Hence, we can say that the function $\varepsilon_{n}(t)$ is negative in the interval $\left(0, t^{*}\right)$, is positive in $\left(t^{*}, T\right)$, is uniformly bounded in its domain, that is, the two-sided inequality

$$
-\left\|l_{n}-h_{n}\right\|<\varepsilon_{n}(t)<\left\|L_{n}-g_{n}\right\|, \quad t \in[0, T]
$$

holds, where $\left\|L_{n}-g_{n}\right\| \gg\left\|l_{n}-h_{n}\right\|$.

3. The inequality

$$
\varepsilon_{n}^{\prime \prime}(0)=2(2 n+1)\left(L_{n}^{\prime}(0)-g_{n}^{\prime}(0)\right)+h_{n}^{\prime \prime}(0) \equiv 2(2 n+1)\left(\tan \beta\left(A_{1}\right)-\tan \alpha\left(A_{1}\right)\right)+h_{n}^{\prime \prime}(0)>0
$$


holds since both terms in the latter sum are positive: at the vertex $A_{1}=A_{1}\left(0, g_{n}(0)\right)$, the substense forms a greater angle $\beta\left(A_{1}\right)$ than the angle $\alpha\left(A_{1}\right)$ between the axis $O t$ and the tangent to the graph of the function $y=g_{n}(t)$ at the point $A_{1}$. Since the graph of the function $y=h_{n}(t), t \in[0, T]$, is concave, it holds $h_{n}^{\prime \prime}(0)>0$.

We have

$$
\varepsilon_{n}^{\prime \prime}(T)=2(2 n+1)\left(l_{n}^{\prime}(T)-h_{n}^{\prime}(T)\right)-g_{n}^{\prime \prime}(T)=2(2 n+1)\left(\tan \beta\left(B_{2}\right)-\tan \alpha\left(B_{2}\right)\right)-g_{n}^{\prime \prime}(T)<0,
$$

which is implied by a similar arguing made in view of the features of the vertex $B_{2}\left(T, h_{n}(T)\right)$ of the graph of the function $h_{n}(t)$.

Thus, the results of Items 1)-3) and additional information on the terms $p_{n}$ and $q_{n}$ of error function (67) obtained by means of differential calculus allow us to state the following:

$$
\tilde{I}_{\varepsilon}(n)=\frac{2}{\pi(2 n+1)}\left(\int_{0}^{t^{*}} \varepsilon_{n}(t) d t+\int_{t^{*}}^{T} \varepsilon_{n}(t) d t\right)>0, \quad n \in \mathbb{N} .
$$

As the result of the above studies (see (64), (66), (69)), we can formulate the following theorem.

Theorem 8. Functional dependence (32) satisfies the following uniform upper bound

$$
I_{1}(n)<\varphi_{4}(n), \quad n \in D_{1}=\mathbb{N},
$$

where the function in the right hand side of the inequality is defined by formula (18).

Remark 6. By identities (36), (64), (66) and notations (17), (18) we have the equivalence

$$
\tilde{I}_{\varepsilon}(n)=I_{2}(n)-\left(\varphi_{3}(n)-\varphi_{4}(n)\right), \quad n \in \mathbb{N} \quad \Leftrightarrow \quad \tilde{I}_{\varepsilon}(n)=I_{2}(n)-\frac{4}{\pi^{2}} \alpha_{n}^{2} \cos \frac{\pi}{4 n+2}, \quad n \in \mathbb{N},
$$

where the right hand side of the latter identity is represented as the difference of two positive functions. Therefore, we can check inequality (69) by estimating integral (33) from above, that is, by checking the inequality

$$
I_{2}(n)>\frac{4}{\pi^{2}} \alpha_{n}^{2} \cos \frac{\pi}{4 n+2}, \quad n \in \mathbb{N} .
$$

Theorems 2, 3, 5, 8 allow us to solve the extremal problem formulated in the introduction.

Theorem 9. The constant $A^{*}=\frac{1}{3}+\frac{2 \sqrt{3}}{\pi}$ solves extremal problem 2, that is,

$$
\min \left\{A \mid L_{n} \leqslant A+\frac{4}{\pi^{2}} \ln n, n \in \mathbb{N}\right\}=A^{*}, \quad A^{*}=\frac{1}{3}+\frac{2 \sqrt{3}}{\pi}=1.435991 \ldots
$$

Proof. Formula (37) for the Lebesgue constant holds immediately in the subdomain $D_{2}=$ $\{3,4,5,6, \ldots\}$ of the set of natural numbers $\mathbb{N}$. We estimate its terms $I_{0}(n), S(n), I_{1}(n)$ from above in the domain $D_{2}$ employing at that relations (27), (57), (70). Then we apply corresponding estimates (13), (16), (22) to the functions $\varphi_{1}(n), \varphi_{2}(n), \varphi_{4}(n), n \in D_{2}$. As a result, we get

$$
\begin{aligned}
L(n) & =I_{0}(n)+S(n)+I_{1}(n)<\varphi_{1}(n)+\varphi_{2}(n)+\frac{4}{\pi^{2}} \ln n+\varphi_{4}(n) \\
& <\max _{n \in D_{2}} \varphi_{1}(n)+\max _{n \in D_{2}} \varphi_{2}(n)+\max _{n \in D_{2}} \varphi_{4}(n)+\frac{4}{\pi^{2}} \ln n \\
< & 0.880440+0.439594+0.093757+\frac{4}{\pi^{2}} \ln n<1.413791+\frac{4}{\pi^{2}} \ln n \\
& \Rightarrow L(n)<1.413791+\frac{4}{\pi^{2}} \ln n \equiv \tilde{\mu}_{3}(n), \quad n \in D_{2} \subset \mathbb{N} .
\end{aligned}
$$


Employing exact values of the Lebesgue constants $L(1), L(2)$ calculated in (39), we check inequality (71) for $n=2$ :

$$
L(2)=1.642188 \ldots, \quad \tilde{\mu}_{3}(2)=1.413791+\frac{4}{\pi^{2}} \ln 2=1.694712 \ldots \quad \Rightarrow \quad L(2)<\tilde{\mu}_{3}(2) .
$$

Therefore, inequality $(71)$ in a wide set $D_{3}=\{2,3,4,5,6, \ldots\}$. As $n=1$, it fails, that is, the inequality $L(1)>\tilde{\mu}_{3}(1)$ holds $(\Leftrightarrow 1.435991 \ldots>1.413791 \ldots)$.

In order to fix this situation, we move the vertex $K(1,1.413791)$ of the graph of the function $y=\tilde{\mu}_{3}(n)$ up to the point $L\left(1, \frac{1}{3}+\frac{2 \sqrt{3}}{\pi}\right)$ parallel along the axis $O n$. At that, the logarithmic function $\tilde{\mu}_{3}(n)$ coincides identically with the function

and the inequalities

$$
y=\tilde{\mu}(n)=\frac{1}{3}+\frac{2 \sqrt{3}}{\pi}+\frac{4}{\pi^{2}} \ln n
$$

$$
L(n)<\tilde{\mu}_{3}(n)<\tilde{\mu}(n), \quad n \in D_{3},
$$

hold. As $n=1$, we have the identity:

$$
L(1)=\tilde{\mu}(1)=\frac{1}{3}+\frac{2 \sqrt{3}}{\pi} .
$$

Therefore, in the entire set of the natural numbers $\left(N \supset D_{3} \supset D_{2}\right)$ the inequality

$$
L(n) \leqslant \frac{1}{3}+\frac{2 \sqrt{3}}{\pi}+\frac{4}{\pi^{2}} \ln n, \quad n \in \mathbb{N},
$$

holds true. The proof is complete.

Remark 7. Theorem 9 and inequalities (61) allow us to make the following conclusion: the error $O_{n} \equiv L_{n}-\frac{4}{\pi^{2}} \ln n$ of the Lebesgue function satisfies the two-sided inequality

$$
1.173198<L_{n}-\frac{4}{\pi^{2}} \ln n \leqslant \frac{1}{3}+\frac{2 \sqrt{3}}{\pi}, \quad n \in \mathbb{N}, \quad \frac{1}{3}+\frac{2 \sqrt{3}}{\pi}=1.435991 \ldots,
$$

in which the upper bound is sharp.

\section{BIBLIOGRAPHY}

1. I.P. Natanson. Constructive function theory. Gostekhizdat, Moscow (1949). [Frederick Ungar Publishing Co., New York (1965).]

2. A. Zygmund. Trigonometric series, Cambridge Univ. Press, Cambridge (1959).

3. A.F. Timan. Theory of approximation of functions of a real variable. Fizmatgiz, Moscow (1960). [Intern. Ser. Monog. Pure Appl. Math. 34. Pergamon Press, Oxford (1963).]

4. V.M. Tikohmirov. Some issues of approximation theory. Moscow State Univ. Publ., Moscow (1976). (in Russian).

5. L. Fejér Lebesguesche konstanten und divergente Fourierreihen // J. Reine Angew. Math. 1910:138, 22-53 (1910).

6. L. Fejér. Sur les singularités de la série de Fourier des fonctions continues // Ann. de l'Éc. Norm. Ser. 3. 28, 63-103 (1911).

7. G. Szegö. Über die Lebesgueschen konstanten bei den Fourierchen reihen // Math. Z. 9:1-2, 163166 (1921).

8. G.H. Watson. The constant of Landau and Lebesgue // Quart. J. Math. Ser. 1. os-1:1, 310-318 (1930).

9. I.P. Kornejchuk. Extremal problems of approximation theory. Nauka, Moscow (1976). (in Russian).

10. G.H. Hardy. Note on Lebesgues constants in the theory of Fourier series // J. London Math. Soc. s1-17:1, 4-13 (1942).

11. S.M. Nikol'skii. On linear methods of summation of Fourier series // Izv. AN SSSR. Ser. Matem. 12:3, 259-278 (1948). (in Russian). 
12. S.B. Stechkin. Some remarks on trigonometric polynomials // Uspekhi Matem. Nauk. 10:1, 159166 (1955). (in Russian).

13. P.V. Galkin. Estimate for Lebesgue constants // Trudy MIAN SSSR. 109, 3-5 (1971). [Proc. Steklov Inst. Math. 109, 1-4 (1971).]

14. N.I. Akhiezer. Lectures on approximation theory. Mir, Moscow (1965).

15. V.V. Zhuk, G.I. Natanson. Trigonometrical Fourier series and elements of approximation theory. Izdat. Leningr. Univ. (1983). (in Russian).

16. G.I. Natanson. An estimate for Lebesgue constants of de la Vallée-Poussin sums // in "Geometric questions in the theory of functions and sets". Kalinin State Univ., Kalinin (1986). (in Russian).

17. S.L. Sandakova. Approximations of functions by Fourier sums over trigonometric orthogonal polynomials. PhD thesis, Ural State Univ. named after A.M. Gorky, Ekaterinburg (2005). (in Russian).

18. V.M. Badkov. Introduction to a unified theory of algebraic and trigonometric orthogonal polynomials. Ural State Univ. Publ., Ekaterinburg (2006). (in Russian).

19. V.P. Motorny, S.V. Goncharov, P.K. Nitiema. On mean convergence of Fourier-Jacobi series // Dopovidi Natsion. Akad. Nauk Ukrainy. 3, 35-55 (2010). (in Russian).

20. V.M. Badkov. Estimates of the Lebesgue function of Fourier sums over trigonometric polynomials orthogonal with a weight not belonging to the spaces $L_{r}(r>1)$ // Trudy IMM UrO RAN. 17:3, 71-82 (2011). [Proc. Steklov Inst. Math. Suppl. 1. 277, 21-32 (2012).]

21. A.Yu. Trynin. Estimates for the Lebesgue functions and the Nevai formula for the sincapproximations of continuous functions on an interval // Sibir. Matem. Zhurn. 48:5, 1155-1166 (2007). [Siber. Math. J. 48:5, 929-938 (2007).]

22. I.A. Shakirov. A complete description of the Lebesgue functions for classical Lagrange interpolation polynomials // Izv. VUZov. Matem. 10, 80-88 (2011). [Russ. Math. 55:10, 70-77 (2011)]

23. I.A. Shakirov. Lebesgue functions corresponding to a family of Lagrange interpolation polynomials // Izv. VUZov. Matem. 7, 77-89 (2013). [Russ. Math. 57:7, 66-76 (2013).]

24. I.A. Shakirov. Influence of the choice of Lagrange interpolation nodes on the exact and approximate values of the Lebesgue constants // Sibir. Matem. Zhurn. 55:6, 1404-1423 (2014). [Siber. Math. J. 55:6, 1144-1160 (2014).]

Iskander Asgatovich Shakirov,

Naberezhnye Chelny State

Pedagogical University,

Nizametdinova str. 28,

423806, Naberezhnye Chelny, Russia

E-mail: iskander@tatngpi.ru 